\title{
Successful Treatment of Necrotizing Fasciitis of the Upper Extremity Caused by Vibrio vulnificus: Two Case Reports and Literature Review
}

Pengchao Guo ( 2515170@zju.edu.cn )

zhejiang university

Nan Li

Zhejiang University School of Medicine Second Affiliated Hospital

Quangfeng Zhao

Zhejiang University School of Medicine Second Affiliated Hospital

Xueqing Hu

Zhejiang University School of Medicine Second Affiliated Hospital

\section{Case Report}

Keywords: Vibrio vulnificus, Necrotizing Fasciitis, emergency debridement surgery, upper limb, bacteraemia, anti-infective drug.

Posted Date: January 29th, 2021

DOl: https://doi.org/10.21203/rs.3.rs-154368/v1

License: (c) (i) This work is licensed under a Creative Commons Attribution 4.0 International License. Read Full License 


\section{Abstract}

Background: Vibrio vulnificus infection patients are more common in China's coastal areas and military activities, but there have been very rare reports successful treatment and limb salvage patients.

Case presentation: We present two cases of patients with the successful treatment of necrotizing fasciitis caused by $V$. vulnificus and review the pathogenetic mechanism, epidemiology, clinical characteristics, and treatment of this infection.

Conclusion: When Vibrio vulnificus patients develop bacteraemia and necrotizing fasciitis symptoms,emergency surgical debridement combined with anti-infection therapy should be considered.

\section{Background}

Vibrio vulnificus grows in water with the appropriate temperature and salinity and is mainly obtained by seafood ingestion or direct contact ${ }^{[1]}$. In susceptible individuals, traumatic infections can be fatal, leading to severe wound infections and even septic shock, which may require amputation ${ }^{[2]}$. The pathogenesis of $V$. vulnificus-associated sepsis is very complex and includes iron uptake, cell damage, adhesion-related proteins and virulence regulation ${ }^{[3]} . V$. vulnificus infection is mainly manifested by clinical events such as primary sepsis and traumatic infection, and signs of multiple organ dysfunction

syndrome (MODS) soon appear ${ }^{[4]}$. In the diagnosis and treatment of this disease, it is important to evaluate these pathogenic bacteria in combination with medical history and bacteriological culture results so that more sensitive antibiotics and timely surgical intervention can be selected to effectively improve the prognosis of patients. In this report, we share a case of the successful diagnosis and treatment of septic shock caused by $V$. vulnificus infection (which occurred at a national first-level trauma center and summarize the typical clinical manifestations and treatment methods.

\section{Case Presentation}

\section{Case 1}

A 46-year-old male with no history of chronic liver disease or cancer was admitted to our emergency department with a diagnosis of severe fever caused by an accidental stab wound that he had sustained on the dorsum of his right hand while handling seafood 2 days before. The patient's right palm became swollen and uncomfortable, and he immediately rushed to the local hospital for treatment. The first doctor empirically applied antibiotics based on the medical history. The exposure time from the first onset was 6 hours, and the patient exhibited ecchymosis and blisters around his right palm and wrist joints. The local hospital only administered antibiotics as conservative treatment and did not perform emergency surgical incision and drainage interventions. The results of high-throughput sequencing of this patient's blood drawn from the local hospital suggested that the source of infection was $V$. vulnificus. The patient was transferred from the local hospital to our hospital for treatment after 17 hours. Physical 
examination revealed that his right upper limb was severely inflamed with ecchymosis and several blisters (Fig. 1a). A preoperative examination was conducted, and the patient was given a third-generation cephalosporin and quinolone as a combination anti-infective drug treatment. The right forearm incision and drainage were performed immediately in the emergency department, but a negative pressure drainage device was not used (Fig. 1b). The patient's vital signs gradually improved on the 7th day. After a series of treatments, the wound did not deteriorate further, nor did it progress to necrotizing fasciitis. After the 24th day, a right hand skin graft was performed. The patient's limb salvage was successful. He recovered and was discharged on the 26th day after the operation.

\section{Case 2}

A 60-year-old female with a history of gout and rheumatic heart disease was admitted to our emergency department with a diagnosis of severe fever with bacteraemia due to a scratch on her right index finger caused by the scales of a crucian carp 12 hours earlier. On the same day, the patient's right hand became swollen and purple in hue, and the little finger of the same hand was swollen with purple and black discoloration. She immediately rushed to our hospital for treatment. The exposure time from the first onset was 12 hours. The initial diagnosis was severe septic shock and right forearm compartment syndrome. Physical examination revealed severe inflammation of the right upper limb with ecchymosis and several blisters (Fig. 2a). The swelling of the right forearm rapidly progressed to the proximal part of the limb, and the blisters expanded and ruptured within 12 hours; the patient's condition then deteriorated rapidly, and septic shock appeared. She underwent acute incision debridement immediately after admission (Fig. 2b). The drainage fluid of the wound during the operation was cultured, and the test result suggested Aeromonas veronii. The patient was transferred to the intensive care unit for treatment after the operation. Treatment measures included anti-infective therapeutics, fluid resuscitation, vasoactive drugs, daily dressing changes, and continuous renal replacement therapy (CRRT). The results of high-throughput sequencing after the first operation indicated that the infectious bacterium was $V$. vulnificus. After a series of treatments, the wound did not improve and instead progressed to necrotizing fasciitis. On the 12th day, the patient underwent wound debridement and expansion along with amputation of the right little finger, and the postoperative wound was treated with a vacuum sealing drainage (VSD)/negative pressure drainage device (Fig. 2c). The patient's condition improved after this operation, and she was transferred to the general ward to continue treatment. A right forearm skin graft was performed on the 24th day, and the patient's limb salvage was successful. She recovered and was discharged on the 41 st day.

\section{Clinical characteristics and treatment}

General information regarding the 2 admitted patients with $V$. vulnificus infection is shown in Table 1 . Disease characteristics are as follows: The patient first manifested with an acute onset, swelling of the affected area, severe pain, and impaired mobility, followed by ecchymosis, tension blisters and even skin necrosis in the affected area. One severely ill patient developed septic shock within 24 hours. In addition to the typical clinical manifestations of the two patients, namely, fever, low blood pressure or symptoms 
of septic shock, and characteristic vesicular lesions, the female patient had a history of rheumatic disease. Laboratory examinations of the two patients revealed that the white blood cell counts; proportion of neutrophils; C-reactive protein, procalcitonin, and blood sugar levels; and other indicators were all elevated. Moreover, haemoglobin, albumin, and platelets were decreased. Incision of the skin of the affected area showed extensive necrosis of the fascial tissue, a large amount of water-like exudate from the irrigated tissue, and no obvious odour.

Table 1

\begin{tabular}{|c|c|c|c|c|c|c|c|}
\hline \multicolumn{8}{|c|}{ General information of the two cases } \\
\hline \multirow[t]{2}{*}{ Case } & \multirow[t]{2}{*}{ Age } & \multirow[t]{2}{*}{ Sex } & \multirow[t]{2}{*}{ History } & \multirow[t]{2}{*}{ Pathogen } & \multirow{2}{*}{$\begin{array}{l}\text { Time of } \\
\text { the first visit }\end{array}$} & \multicolumn{2}{|l|}{ Bacterial culture } \\
\hline & & & & & & Wound secretions & Blood \\
\hline 1 & 46 & male & no & \multirow[t]{2}{*}{ handling of fish } & 6 hours & + & \multirow[t]{2}{*}{$t^{\mathrm{c}}$} \\
\hline 2 & 60 & female & yes $^{a}$ & & 12 hours & $+b$ & \\
\hline \multicolumn{8}{|c|}{ a: Female with a history of rheumatic immune disease. } \\
\hline \multicolumn{8}{|c|}{ b: The results from the culture of wound secretions suggested Aeromonas veronii. } \\
\hline \multicolumn{8}{|c|}{ c: The results of blood-based NGS indicated Vibrio vulnificus. } \\
\hline
\end{tabular}

\section{Results of aetiological examination}

V. vulnificus was cultured in the tissue fluid and blood from the first affected area of the female patient. The male patient was referred with next-generation sequencing (NGS) ${ }^{[5]}$ results reported at the local hospital as indicating $V$. vulnificus. After the two patients were transferred to our hospital for multiple debridement procedures and combined antibiotic treatment, the final culture result was negative. The results of the drug sensitivity test showed that $V$. vulnificus was sensitive to most antibiotics, among which third-generation cephalosporins and quinolones had the lowest minimum inhibitory concentration (MIC) value and the strongest in vitro sensitivity.

\section{Treatment (Table 2)}

The patients were actively rehydrated to correct shock. According to the NGS results combined with treatment guidelines, the administered antibiotics comprised cefotaxime combined with quinolone therapy ${ }^{[6]}$. The two patients underwent emergency debridement immediately after diagnosis; in addition, debridement operations were repeated many times, and wound sealing and negative pressure drainage were performed. 
Table 2

\section{Two cases of the treatment of patients infected with Vibrio vulnificus}

\begin{tabular}{|llllll|}
\hline Case & Antibiotics & $\begin{array}{l}\text { Operation } \\
\text { time }\end{array}$ & Surgical approach & $\begin{array}{l}\text { CRRT } \\
\text { (ICU) }\end{array}$ & $\begin{array}{l}\text { Hospital } \\
\text { Day(HD) }\end{array}$ \\
\hline $\begin{array}{l}\text { 1: } \\
\text { male }\end{array}$ & $\begin{array}{l}\text { Piperacillin sodium } \\
\text { Tazobactam sodium + } \\
\text { Levofloxacin }\end{array}$ & 3-times & $\begin{array}{l}\text { Left upper limb debridement } \\
\text { +vacuum negative pressure } \\
\text { closed suction }\end{array}$ & no & 28 days \\
$\begin{array}{l}\text { 2: } \\
\text { female }\end{array}$ & $\begin{array}{l}\text { Vancomycin }+ \\
\text { Meropenem + } \\
\text { Moxifloxacin }\end{array}$ & 5-times & $\begin{array}{l}\text { Right upper limb } \\
\text { debridement + vacuum } \\
\text { negative pressure closed } \\
\text { suction }\end{array}$ & yes & 41 days \\
\hline $\begin{array}{l}\text { All patients underwent more than 2 operations, and they were treated with a combination of surgery } \\
\text { and antibiotics. }\end{array}$ & & & & \\
\hline
\end{tabular}

\section{Clinical outcome}

Both patients improved during the treatment process and finally succeeded in protecting their limbs. They were cured and discharged without major amputation.

\section{Discussion}

\section{Pathogenetic mechanism}

V. vulnificus is a mesophilic, halophilic, gram-negative bacterium that lives in the ocean. It belongs to the same genus as V. cholerae and V. parahaemolyticus ${ }^{[7]}$. In 1970, ROLAND first reported gangrene and endotoxic shock of the calf caused by $V$. vulnificus infection ${ }^{[8]}$. In 1979, FARMER named the organism Vibrio vulnificus ${ }^{[9]}$. The pathogenic mechanism of $V$. vulnificus is of great significance to the prevention and treatment of infection by this bacterium ${ }^{[10]}$. At present, research on pathogenic mechanisms mainly focuses on host defence, cytotoxicity, bacterial motility, adhesion-related proteins, virulence regulation, and biofilm formation.

1.1 Host defences include capsular polysaccharides, acid neutralization and iron overload in the body. Capsular polysaccharide (CPS) is produced by V. vulnificus and secreted to the outside of the cell, covering the external immunogenic structure of the bacteria, not only shielding it against the opsonin function of the complement system and the phagocytosis of macrophages but also helping the bacteria escape inherent immune surveillance, which can also determine the morphology of bacterial colonies and regulate the formation of biofilms ${ }^{[11]}$. Acid neutralization means that $V$. vulnificus can resist gastric acid by increasing the expression of lysine decarboxylase and the expression of manganese superoxide dismutase in a low $\mathrm{pH}$ environment in the stomach to achieve acid neutralization ${ }^{[12]} . V$. vulnificus obtains 
iron in the blood through various iron uptake systems and then grows and proliferates. Serum iron levels can indirectly reflect the infection of the body with $V$. vulnificus.

1.2 Cytotoxicity involves lipopolysaccharide (LPS), cytolysin and repeats-in-toxin A1 (RtxA1). LPS may play a role in cytotoxicity by affecting the activity of nitric oxide synthase (NOS) in the body; LPS can also play an adhesive role to promote the formation of biofilms, causing the infected body to develop endotoxin shock and sepsis ${ }^{[13]}$. Cytolysin is an exotoxin encoded by the vvhA gene and is secreted outside of the cell. It exerts cytotoxicity that causes cell apoptosis and can destroy red blood cells to release iron into the blood ${ }^{[14]}$. RtxA1 is capable of mediating cell death and tissue necrosis after contact with bacterial cells, thereby increasing the expression of the RtxA1 gene and inducing cytotoxicity and the destruction of intestinal epithelial cells and intestinal microvilli ${ }^{[15]}$.

1.3 Bacterial motility involves flagella and fimbriae. $V$. vulnificus has one flagellum, which is encoded by 6 flagellum genes (flaA, flaB, flaC, flaD, flaE, flaF), of which flaB, flaD and flaC play a major role ${ }^{[16]}$. Flagella are related to the motility, adhesion, cytotoxicity, biofilm formation and invasion of bacteria ${ }^{[17]} . \mathrm{V}$. vulnificus fimbriae were first discovered by GANDER under an electron microscope in $1989{ }^{[18]}$. The fimbriae are related to bacterial invasion, adhesion and pathogenicity.

1.4 Extracellular proteases and adhesion-related proteins: Outer membrane protein $\mathrm{U}(\mathrm{OmpU})$ can bind to fibronectin, the main component of the mammalian extracellular matrix and is related to the adhesion function of bacteria ${ }^{[19]}$. Membrane-bound lipoprotein A (IIpA) plays a role in adhesion and immunogenicity. The extracellular protease (ECPase) of $V$. vulnificus can yield a variety of ECPases that exert cytotoxic effects, including metalloprotease (Mpase), chondroitinase (ChSase), and hyaluronidase (HAase) ${ }^{[20]}$.

1.5 Toxicity regulation includes quorum sensing (QS), total virulence regulators and haemolysin $\mathrm{U}$. Bacteria can synthesize and release a kind of autoinducer (Al) that regulates many of their biological behaviours, thus causing group-sensing effects. In $V$. vulnificus, the synthesis of CPS and fimbriae is regulated by the QS system ${ }^{[21]}$. The global virulence regulator CAMP-CAMP receptor protein (CRP) can bind to DNA and affect gene expression, including cytolysin, metalloproteinase and iron uptake systems, which are all regulated by the CRP system. Another virulence regulator, AphB, has a wide range of functions, including acid neutralization, motility, adhesion and pathogenicity ${ }^{[22]}$. Haemolysin $U(\mathrm{Hly} U)$ is the main regulator of $V$. vulnificus toxicity and can regulate the expression of the $r \mathrm{txA} 1, \mathrm{vvhA}$ and $\mathrm{vvpE}$ genes.

1.6 Biofilm formation. The biomembrane composed of CPS, exopolysaccharides (EPS) and LPS can counter the effects of drugs and the host immune system ${ }^{[23]}$.

\section{Epidemiology}


V. vulnificus exists as a free-living bacterium inhabiting estuarine or marine environments. Traditionally, three biotypes have been recognized: biotype 1, which accounts for almost all human infections; biotype 2 , which consists primarily of eel pathogens; and biotype 3 , an apparent hybrid of biotypes 1 and 2 that has been described in tilapia-associated wound infections associated with aquaculture in Israel [24]. Wound infections most often occur in the setting of handling seafood and generally result from exposure of a wound to salt or brackish water containing the organism ${ }^{[25]}$. Individuals with the following conditions are at increased risk for serious infection with $V$. vulnificus ${ }^{[26]}$ : Alcoholic cirrhosis, Underlying liver disease including cirrhosis and chronic hepatitis, Alcohol abuse without documented liver disease, Hereditary haemochromatosis, Chronic diseases such as diabetes mellitus, rheumatoid arthritis, thalassemia major, chronic renal failure, and lymphoma. Interestingly, men, and particularly older men, appear to be at much greater risk for serious infection than women ${ }^{[27]}$. Our motherland has a vast population and a large group of patients with liver cirrhosis. The eastern coast of China has a coastline of 32,000 kilometres and a sea area of nearly 3 million square kilometres. The development of fishery operations and military activities in coastal areas makes this region a high-risk area for $V$. vulnificus infection.

\section{Clinical characteristics}

The trend of $V$. vulnificus infection increases with climate warming, ocean activities, and the presence of high-risk factors such as alcohol, liver disease, systemic disease, and diabetes ${ }^{\text {[28] }}$. Traumatic bacterial infection is mainly manifested in the following clinical subtypes: traumatic infection, primary sepsis, gastroenteritis and typical clinical features. Injuries with mild symptoms may result from infection with $V$. vulnificus, which may cause cellulitis with mild clinical symptoms ${ }^{[29]}$. However, in high-risk individuals (such as the female patient reported in this article) who have a history of rheumatic disease, the infection may spread quickly, causing severe myositis and necrotizing fasciitis (Fig. 1). This pathogen enters the gastrointestinal tract through contact with open wounds, thereby entering the blood system and then causing sepsis. The main disease manifestations include acute fever, chills, shock, and skin and muscle damage. People with gastrointestinal infections exhibit symptoms of acute gastroenteritis, including diarrhoea, nausea, vomiting, abdominal pain, and difficulty breathing ${ }^{[30]}$. Typical skin and muscle damage includes local or flaky erythema and ecchymosis, bloody vesicles with exudation, necrosis and cellulitis, necrotizing fasciitis, and muscle inflammation ${ }^{[31]}$. In the case of body infection caused by contact with seawater, skin and muscle damage rapidly progresses to necrosis and leads to severe sepsis, which may lead to life-threatening conditions ${ }^{[32]}$. Patients usually have underlying liver disease, alcoholism, hereditary pigmentation diseases, or the chronic diseases mentioned above, which can cause primary sepsis. Approximately one-third of patients with primary sepsis will have shock or low blood pressure within 12 hours after going to the hospital.

Three-quarters of patients exhibit unique blister lesions (Fig. 2). The infection causes complications such as reduced blood circulation, diffuse intravascular coagulation, and disorders of the digestive tract. Primary $V$. vulnificus sepsis is an extremely serious disease with a high mortality rate. 


\section{Treatment}

The pathogenic diagnosis of the two patients reported in this article relied on the results of NGS. With its high-output and high-resolution characteristics, NGS not only provides us with rich genetic information but also greatly shortens the cost and time of sequencing and provides more accurate clinical guidance for the diagnosis of clinical diseases. The US Centers for Disease Control (CDC) recommended three generations of cephalosporins combined with tetracyclines as the recommended treatment for $V$. vulnificus infection. Patients with mild to moderate infections are usually treated with antibacterial drugs for 5-7 days. If patients with mild infections have no serious underlying diseases, mild wound infections usually respond well to topical treatment and intravenous infusion of antibacterial drugs (such as cephalosporins combined with tetracyclines or cephalosporins combined with quinolones). The length of treatment depends on the severity of the initial infection and the clinical response. Treatment of high-risk patients with severe infection: Patients who are presumed to be diagnosed with $V$. vulnificus sepsis should start antibacterial drugs and incision debridement as soon as possible, and when necessary, amputation plays a significant role in improving the prognosis of patients with $V$. vulnificus infection ${ }^{[33]}$. For patients with severe haemodynamic instability, a low platelet count and severe coagulopathy,

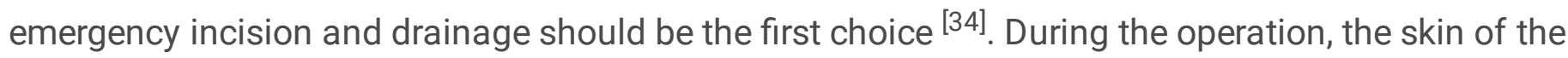
infected limb should be incised, the subcutaneous tissue should be bluntly separated, and the fascia should be exposed until the muscle membrane is formed under local anaesthesia or intravenous pain relief. The length and direction of the incision are determined according to the patient's coagulation function, skin tension, and degree of disease. After the operation, gauze soaked in iodophor and sulfamethazine solution is used for external application to facilitate dressing changes and timely assessment of the wound surface. Active treatment in the intensive care unit where possible, along with early CRRT, can help MODS patients by removing endotoxin and inflammatory mediators. Patients with acute lung injury or acute respiratory distress syndrome (ARDS) should be given non-invasive or invasive mechanical ventilation to minimize the possible consequences of low pressure and septic shock and to reduce the risk of multiple organ system failure. The affected limb should be amputated to save a patient's life if the muscle necrosis is too severe to mend.

\section{Conclusion}

Combined with the exchange of clinical cases and experience, a clear history of exposure, a rapidly deteriorating general state and local typical bloody bullous lesions are important evidence for the diagnosis of sepsis caused by $V$. vulnificus infection. Most patients develop hypotension or shock, rapid

development of symptoms and MODS within 24-48 hours, and the mortality rate exceeds 50 percent ${ }^{\text {[35] }}$. Although the culture of pathogenic bacteria is delayed, it is difficult to recognize the infection of $V$. vulnificus in time. When conditions permit, the NGS test can obtain the result of pathogenic bacteria faster and more accurately. Experienced surgeons should assess whether emergency surgery is needed to treat and control the infection as soon as possible because early surgical intervention can improve the prognosis of patients with $V$. vulnificus. We recommend the combination of anti-infection therapy and 
surgical debridement as soon as possible, and surgical debridement should be performed as soon as possible after the diagnosis is confirmed. Early surgical debridement of our two patients improved their treatment outcome. Standard surgical debridement, incision and drainage can quickly alleviate the patient's pain, reduce the loss of various coagulation factors in the early stage of infection, reduce the patient's bleeding risk, and improve the patient's disease tolerance. Wound sealing and negative pressure drainage technology can help limit infection, drain exudates, and reduce oedema ${ }^{[36]}$. Whether the patient limb is amputated is based on the assessment of the patient's wound because early amputation may help completely remove the source of infection, block the chain of bacterial transmission, and enable the patient to enter the "improvement period" as soon as possible, which may benefit him/her. In view of the dangerous condition of $V$. vulnificus infection and the high mortality rate of certain populations, sufficient attention should be paid.

\section{Abbreviations}

NGS: next-generation sequencing ; CT: Computed tomography; HD: Hospital day; CRRT: continuous renal replacement therapy.

\section{Declarations}

\section{Acknowledgements}

We gratefully acknowledge the intense individual effort and support from many sources to make this study possible, as well as the contributions of plasma donors.

\section{Authors' contributions}

LN and GPC have full access to all the data in the study and take responsibility for the integrity of the data and the accuracy of the data analysis. GPC and ZGF conducted concept and design. LN, ZGF and HXQ performed the data collection, analysis and interpretation. LN and GPC drafted the manuscript. All authors read and approved the final manuscript.

\section{Funding}

Not applicable.

\section{Availability of data and materials}

Not applicable.

\section{Ethics approval and consent to participate}

This study was approved by the Medical Ethical Committee of zhejiang university medical of second hospital with the participants' written informed consent (ZEYYMECT:L20205487 L011). 


\section{Consent for publication}

Not applicable.

\section{Competing interests}

The authors declare that they have no competing interests.

\section{Author details:National trauma center:Level 1}

Nan Li, Xueqing Hu, Plastic surgery department, Second Affiliated Hospital of Zhejiang University School of Medicine, Jiefang street 88 ,Hangzhou, china

Guo Pengchao, Guangfeng Zhao, Emergency department, Second Affiliated Hospital of Zhejiang University School of Medicine, Jiefang street 88,Hangzhou, China.

\section{References}

1. Blake PA, Merson MH, Weaver RE. et al. Disease caused by a marine Vibrio. Clinical characteristics and epidemiology. N Engl J Med. 1979;300:1.

2. Kumamoto KS, Vukich DJ. Clinical infections of Vibrio vulnificus: a case report and review of the literature. J Emerg Med. 1998;16(1):61-6. 10.1016/s0736-4679(97)00230-8. ., ). DOI.

3. Chiang SR, Chuang YC. Vibrio vulnificus infection: clinical manifestations, pathogenesis, and antimicrobial therapy[J]. J Microbiol Immunol Infect. 2003;36(2):81-8.

4. Heng SP, Letchumanan V, Deng CY, Ab Mutalib NS, Khan TM, Chuah LH. et al. Vibrio vulnificus: an environmental and clinical burden. Front Microbiol. 2017;8:997.

5. Tycko Josh DelRosso, Nicole H, Gaelen T, Aradhana B, Abhimanyu M, Aditya. et al. High-Throughput Discovery and Characterization of Human Transcriptional Effectors. Cell. 2020;183:1-16.

6. Dechet AM, Yu PA, Koram N, Painter J. Nonfoodborne Vibrio infections: an important cause of morbidity and mortality in the United States, 1997-2006. Clin Infect Dis. 2008;46:970.

7. OLIVER JD. The biology of vibrio vulnificus[J]. Microbiol Spectr. 2015;3(3):1-10.

8. ROLAND FP. Leg gangrene and endotoxin shock due to vibrio parahaemolyticus an infection acquired in New England coastal waters[J]. N Engl J Med. 1970;282(23):1306.

9. FARMER JJ. Vibrio ("Beneckea") vulnificus, the bacterium associated with sepsis, septicaemia, and the sea[J]. Lancet. 1979;2(8148):903.

10. Arezes João J, Grace G, Victoria VErika, Piotr R, Gulig Paul A. et al. Hepcidin-induced hypoferremia is a critical host defense mechanism against the siderophilic bacterium Vibrio vulnificus. Cell Host Microbe. 2015;17(1):47-57.

11. Pettis Gregg S, Mukerji Aheli SVibrio. vulnificusStructure, Function, and Regulation of the Essential Virulence Factor Capsular Polysaccharide of. Int J Mol Sci. 2020;21(3259):1-13. 
12. Herrera Alfa, Satchell Karla JF. VibrioCross-Kingdom Activation of Toxins by ADP-Ribosylation Factor Family GTPases. J Bacteriol. 2020;202:1-33.

13. Kohei Yamazaki, Takashige KASHIMOTO, Kaho Matsuda M, Yamasaki Mlo, Morita T, Kado. et al. Identification of Essential Genes of for Establishment of Wound Infection by Signature-Tagged Mutagenesis. Front Microbiol. 2019;10:123.

14. Yuan Yuan,Feng Zihan,Wang Jinglin. Vibrio vulnificus Hemolysin: Biological Activity, Regulation of Expression, and Role in Pathogenesis. Front Immunol. 2020;11:599439.

15. Mukherjee Debadrita P, Aritrika C, Devlina. et al. Identification of the target DNA sequence and characterization of DNA binding features of $\mathrm{HlyU}$, and suggestion of a redox switch for hlyA expression in the human pathogen Vibrio cholerae from in silico studies. Nucleic Acids Res. 2015;43:1407-17.

16. Duong-Nu TM, Jeong K, Hong SH, Nguyen HV, Ngo VH, Min JJ. et al. All Three TonB Systems Are Required for Vibrio vulnificus CMCP6 Tissue Invasiveness by Controlling Flagellum Expression. Infect Immun. 2016;84:254-65.

17. Song HC, Kang YH, Zhang DX, Chen L, Qian AD, Shan XF. et al. Great effect of porin(aha) in bacterial adhesion and virulence regulation in Aeromonas veronii. Microb Pathog. 2019;126:269-78.

18. Gander RM, LaRocco MT. Detection of piluslike structures on clinical and environmental isolates of Vibrio vulnificus. J Clin Microbiol. 1989;27(5):1015-21.

19. Hang W, Wei Y, Guoying S. et al. Protein transduction domain of transactivating transcriptional activator fused to outer membrane protein $\mathrm{K}$ of Vibrio parahaemolyticus to vaccinate marbled eels (Anguilla marmorata) confers protection against mortality caused by V. parahaemolyticus. Microb Biotechnol. 2015;8:673-80.

20. Lee SJ, Jung YH, OH SY. et al. Vibrio vulnificus VvpE inhibits mucin 2 expression by hypermethylation via lipid raft-mediated ROS signaling in intestinal epithelial cells. Cell Death Dis. 2015;6:e1787.

21. Kim SM, Park JH, Lee HS, Kim WB, Ryu JM, Han HJ. et al. ChIP-seq analysis of the LuxR-type regulator $\mathrm{VjbR}$ reveals novel insights into the Brucella virulence gene expression network. Nucleic Acids Res. 2017;45:5757-69.

22. Lee YM, Park JP, Jung YH, Lee HJ, Kim JS, Choi GE. et al. Melatonin restores Muc2 depletion induced by $V$. vulnificus VvpM via melatonin receptor 2 coupling with Gaq.[J]J Biomed Sci. 2020;27:21.

23. Xu J, Danehy R, Cai H, Ao Z, Pu M, Nusawardhana A. et al. Microneedle Patch-Mediated Treatment of Bacterial Biofilms ACS Appl Mater Interfaces. 2019;11:14640-6.

24. Broza YY, Danin-Poleg Y, Lerner L, Valinsky L, Broza M, Kashi Y. et al. Epidemiologic study of Vibrio vulnificus infections by using variable number tandem repeats. Emerg Infect Dis. 2009;15:1282-5.

25. Dechet AM, Yu PA, Koram N, Painter J. Nonfoodborne Vibrio infections: an important cause of morbidity and mortality in the United States, 1997-2006. Clin Infect Dis. 2008;46:970-6.

26. Falcon LM, Pham L. Images in clinical medicine. Hemorrhagic cellulitis after consumption of raw oysters. N Engl J Med. 2005;353:1604. 
27. Lee SH, Chung BH, Lee WC. Retrospective analysis of epidemiological aspects of Vibrio vulnificus infections in Korea in 2001-2010. Jpn J Infect Dis. 2013;66:331-3.

28. Hwang J, Kim BS, Jang SY, Lim JG, You DJ, Jung HS. et al. Structural insights into the regulation of sialic acid catabolism by the Vibrio vulnificus transcriptional repressor NanR. Proc Natl Acad Sci USA. 2013;110:E2829-37.

29. Kim CM, Park YJ, Shin SH. A widespread deferoxamine-mediated iron-uptake system in Vibrio vulnifificus. Infect Dis. 2007;196:1537-45.

30. Johnston JM, Becker SF, McFarland LM. Gastroenteritis in patients with stool isolates of Vibrio vulnifificus. Am J Med. 1986;80:336.

31. Mead PS, Slutsker L, Dietz V, McCaig LF, Bresee JS, Shapiro C. Food-related illness and death in the United States.[J].Emerg Infect Dis, 1999, 5: 607-25.

32. Kim JY, Joo YS, Lee S, Lee JY, Park JT, Han SH. et al. Septicemia, necrotizing fasciitis, and peritonitis due to Vibrio vulnificus treated with early use of polymyxin $B$ hemoperfusion in a patient undergoing CAPD: a case report. BMC Nephrol. 2020;21:127.

33. Baker-Austin C, Oliver JD, Alam M, Ali A, Waldor MK, Qadri F. et al. Vibrio spp. infections. Nat Rev Dis Primers. 2018;4:8.

34. Yu W, Shen X, Pan H, Xiao T, Shen P, Xiao Y. Clinical features and treatment of patients with Vibrio vulnificus infection. Int J Infect Dis. 2017;59:1-6.

35. Yun NR, Kim DM, Lee J, Han MA. pH level as a marker for predicting death among patients with Vibrio vulnificus infection, South Korea, 2000-2011. Emerg Infect Dis. 2015;21:259-64.

36. Kim SE, Shin SU, Oh TH, Kim UJ, Darboe KS, Kang SJ, et al. Outcomes of Third-Generation Cephalosporin Plus Ciprofloxacin or Doxycycline Therapy in Patients with Vibrio vulnificus Septicemia: A Propensity Score-Matched Analysis. PLoS Negl Trop Dis. 2019;13:e0007478.

\section{Figures}




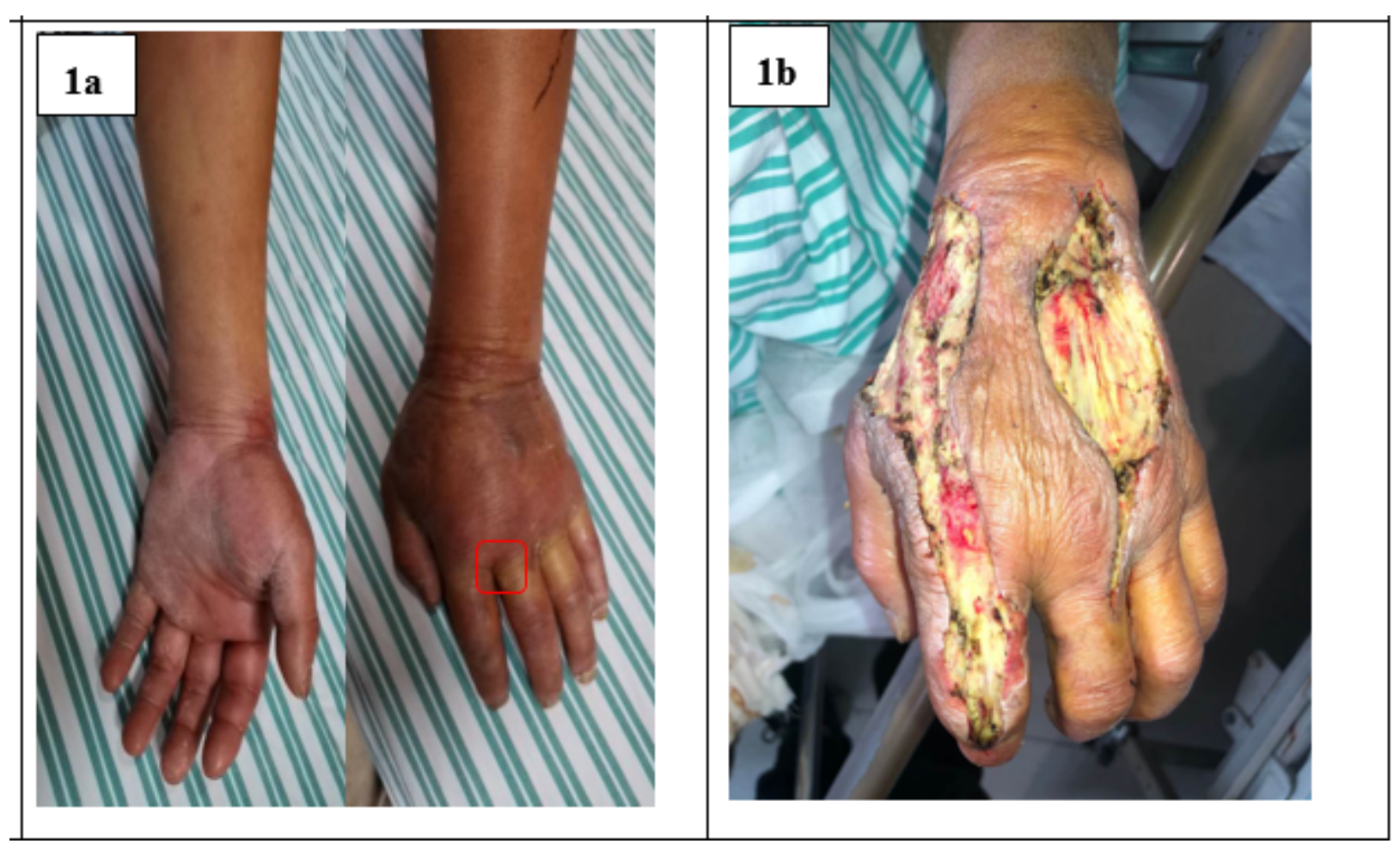

\section{Figure 1}

Surgical photographs of patient, Case 1 male: a: Preoperative, b: Postoperative. The red mark in the picture is the location of the patient's wound injury. 


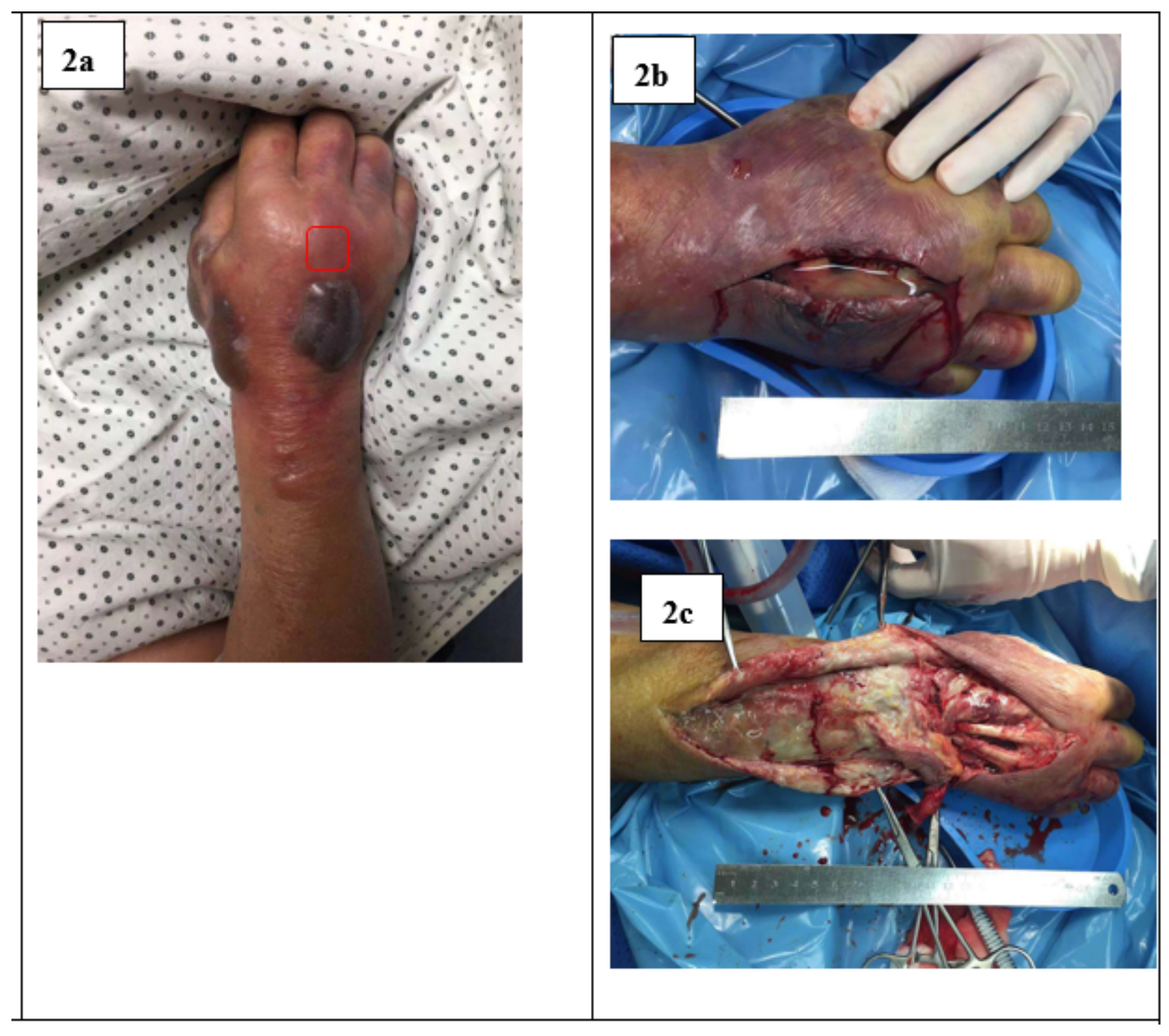

Figure 2

Surgical photographs of patient, Case 2 female: a: Preoperative, b: Picture of the patient's first emergency debridement surgery. c: Picture of the patient's second emergency debridement surgery. The red mark in the picture is the location of the patient's wound injury. 